\title{
Heat capacity of methane-krypton solid solutions. Conversion effect
}

\author{
I. Ya. Minchina, V. G. Manzhelii, M. I. Bagatskii, O. V. Sklyar, \\ D. A. Mashchenko, and M. A. Pokhodenko \\ B. Verkin Institute for Low Temperature Physics and Engineering of the National Academy of Sciences of Ukraine, \\ 47 Lenin Ave., Kharkov 61103, Ukraine \\ E-mail: bagatskii@ilt.kharkov.ua
}

Received February 9, 2001

\begin{abstract}
The heat capacity of $\mathrm{Kr}-n \mathrm{CH}_{4}$ solid solutions with the concentrations $n=1 ; 5 ; 10 \%$ and of the solid solution $\mathrm{Kr}-1 \% \mathrm{CH}_{4}-0.2 \% \mathrm{O}_{2}$ has been studied at $0.7-8 \mathrm{~K}$. The contributions of $\mathrm{C}_{\mathrm{rot}}$ to the heat capacity of the solutions caused by the rotation of the $\mathrm{CH}_{4}$ molecules are estimated. The deviations of the measured $C_{\text {rot }}$ from the values corresponding to the equilibrium distribution of the nuclear spin $\mathrm{CH}_{4}$ modifications are dependent on the correlation between the characteristic times of conversion and of the calorimetric experiment. The effects of temperature, $\mathrm{O}_{2}$ impurities, and $\mathrm{CH}_{4}$ clusters upon the conversion rate are studied. It is shown that the hybrid mechanism of conversion proposed by Berlinsky and Nijman, which takes into account both intramolecular and intermolecular interactions of the proton spins, is predominant.
\end{abstract}

PACS: 65.40.+g

\section{Introduction}

This work is concerned with a calorimetric investigation of the quantum behavior of $\mathrm{CH}_{4}$ rotators in a crystal field of cubic symmetry. The degree of the quantum effect in the rotational motion can be characterized by the parameter $\lambda_{\varphi}=\hbar / \sqrt{I \varepsilon_{\varphi}}$, where $I$ is the moment of inertia of the molecule, and $\varepsilon_{\varphi}$ is the energy barrier that the molecule has to overcome when its orientation changes. The higher $\lambda_{\varphi}$ is the stronger the quantum effects are in the behavior of the rotator. The moment of inertia $I$ is quite small because the $\mathrm{CH}_{4}$ molecule has light hydrogen atoms at its periphery. In matrices of solidified inert gases, methane molecules are surrounded by spherically symmetric atoms. Under this condition, the magnitude of $\varepsilon_{\varphi}$ is also quite low (several tens of kelvin [1]). Solutions of methane in matrices of solid inert gases with the fcc structure are therefore most suitable for answering the questions of this study. The spectrum of a quantum rotator in a crystal field is close to that of a free rotator. As a consequence, the spectra of different nuclear spin modifications of a rotator differ considerably [2], and their mutual transformation (conversion) should be taken into account. Solid $\mathrm{Kr}$ has been chosen as a matrix because, of all the inert gases, only $\mathrm{Kr}$ has Lennard-Jones potential parameters very close to those of $\mathrm{CH}_{4}$. As a result, the solubility of $\mathrm{CH}_{4}$ in solid $\mathrm{Kr}$ is as high as $80 \%$ at low temperatures. This offers an opportunity to investigate not only the behavior of isolated rotators but their interaction as well. Since the low concentration case more readily lends itself to interpretation, we considered it reasonable to use $\mathrm{Kr}-\mathrm{CH}_{4}$ solutions with $\mathrm{CH}_{4}$ concentrations of $1-10 \%$. The quantum behavior of rotators shows up at low temperatures. In addition, the relative contribution of the rotator subsystem to the heat capacity of $\mathrm{Kr}-\mathrm{CH}_{4}$ solutions decreases as the temperature rises. The temperature interval of this study was therefore bounded from above by $8 \mathrm{~K}$.

We should mention some problems which are much assisted by studies of the heat capacity of solid $\mathrm{CH}_{4}-\mathrm{Kr}$ solutions at low (helium) temperatures.

\section{Mechanism of mutual transformation of nuclear spin $\mathrm{CH}_{4}$ modifications (conversion mechanism)}

It is known that $\mathrm{CH}_{4}$ molecules can come in three modifications having different mutual proton spin orientations and rotational energy spectra. 


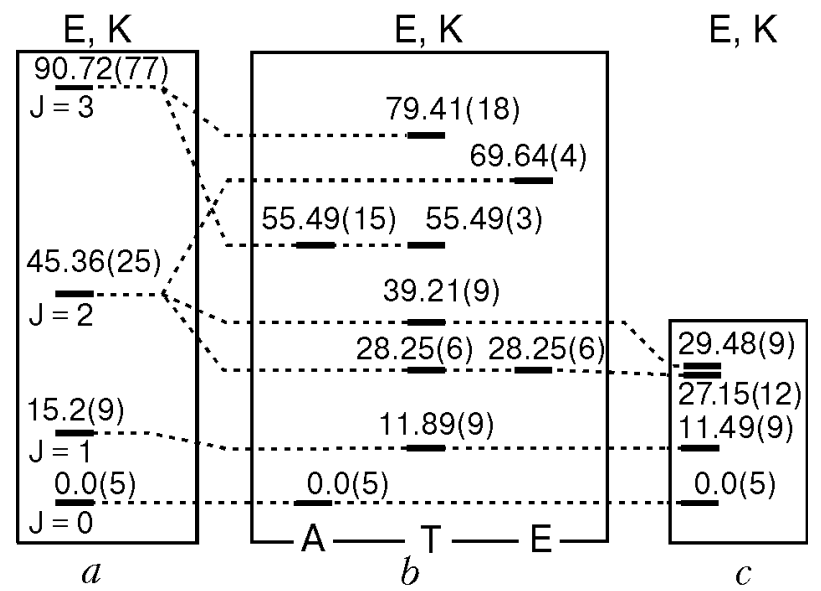

Fig. 1. Rotational energy spectra of the methane molecule: $a-$ free rotator [3]; $b, c-$ hindered $\mathrm{CH}_{4}$ rotator in the crystal $\mathrm{Kr}$ field ( $b$ - calculation [4], $c-$ experimental [5]). $J-$ rotational quantum number, $R$ - energy (degeneracy levels are in brackets to the right).

These $\mathrm{CH}_{4}$ modifications, specified as $A, T, E$, have the total nuclear spins $2,1,0$, respectively. Their rotational spectra (the low-energy region) are shown in Fig. 1 for a free rotator and a rotator in a $\mathrm{Kr}$ matrix.

The $A$ modification has the lowest energy of the ground state. Owing to conversion, the modifications are in equilibrium at different temperatures. The temperature dependence of the equilibrium $A$, $T$, and $E$ concentrations of free $\mathrm{CH}_{4}$ rotators is shown in Fig. 2 [2]. In the high temperature limit the concentration ratio $x_{A}: x_{T}: x_{E}$ is 5:9:2.

Two factors are responsible for the rate of conversion - the interaction of the proton spins and the possibility for the phonons to gain energy from the transitions between different rotational states. Since the spacing of the protons is quite small within the molecule, the intramolecular spin interaction is an order of magnitude stronger than the spin interaction between the neighboring molecules. The intramolecular spin interaction is, however, only weakly connected with the translational vibrations of the lattice, and this considerably impedes conversion. According to Nijman and Berlinsky [6], a hybrid mechanism of conversion predominates in solid $\mathrm{CH}_{4}$ at low temperatures: the intramolecular interaction mixes the nuclear spin states, and the intermolecular interaction induces transitions between the energy levels, which are accompanied by emission of phonons. $\mathrm{CH}_{4}-\mathrm{Kr}$ solid solutions are very suitable to test the Nijman and Berlinsky model. The conversion of isolated $\mathrm{CH}_{4}$ molecules can be studied on weak solutions. At increasing

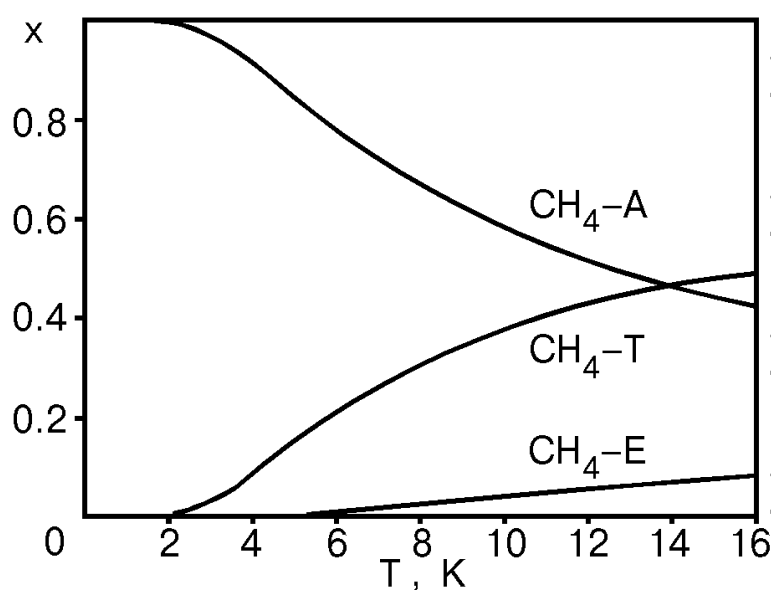

Fig. 2. The temperature dependence of the equilibrium $A, T$, and $E$ concentrations of free $\mathrm{CH}_{4}$ rotators [2].

concentrations, the number of clusters of $\mathrm{CH}_{4}$ molecules increases rapidly, and they trigger the hybrid mechanism of conversion. The heat capacity of the $\mathrm{CH}_{4}$ subsystem is very dependent on the $A, T$, and $E$ concentrations achieved by the time of measurement and on whether their concentration ratio has a chance to change during a single measurement run. It follows from the above consideration that we can derive information about conversion from the measured heat capacity.

To avoid misunderstanding, it is appropriate to note here that our term «heat capacity» has the meaning of the derivative of the heat transferred to the system with respect to temperature, irrespective of whether the system is at equilibrium or not.

It is also interesting to investigate how small amounts of paramagnetic impurities can influence the heat capacity of diluted $\mathrm{CH}_{4}-\mathrm{Kr}$ solid solutions. If the hybrid mechanism prevails and no paramagnetic impurity is present in the $\mathrm{Kr}-\mathrm{CH}_{4}$ solution, during the time of experiment the conversion will mainly occur in the clusters. The addition of a paramagnetic impurity will increase strongly the rate of conversion in the isolated $\mathrm{CH}_{4}$ molecules and hence the heat capacity $C_{\text {rot }}$ of the rotational subsystem. This problem can also be tackled in the context of heat capacity studies.

\section{Dynamics of quantum rotators in a crystal field}

The heat capacity $C_{V}$ of the system is determined by its energy levels. Our heat capacities $C_{P}(P=0)$ estimated at a constant pressure coincide, within the experimental error, with the values $C_{V}$ obtained at a constant volume. The inverse problem of obtaining the energy spectrum from heat capacity [7] can be solved uniquely only for the low-temperature heat capacity, which is determined by a small num- 
ber of excited levels in the spectrum. The first excited state of the rotator subsystem estimated from the heat capacity can sometimes surpass the spectroscopic data in accuracy [8].

The high solubility of $\mathrm{CH}_{4}$ in solid $\mathrm{Kr}[9,10]$ may provide more evidence about how the octupole rotator interaction influences the low-energy part of the spectrum of the rotator subsystems. The multipolar moment of quantum rotators (unlike the classical ones) is dependent on the state of the rotator. In the ground state the $\mathrm{CH}_{4}$ molecule has the smallest [3] octupole moment. As the temperature of the $\mathrm{Kr}-\mathrm{CH}_{4}$ solution rises, the occupancy of the excited states of the $\mathrm{CH}_{4}$ rotators increases, and so do the octupole moment of the $\mathrm{CH}_{4}$ molecules and their interaction. Thus, the interaction between the $\mathrm{CH}_{4}$ molecules changes with temperature, which should manifest itself in the behavior of the heat capacity of the rotator subsystem.

\section{Octupole glasses in $\mathrm{Kr}-n \mathrm{CH}_{4}$ solutions: existence and features}

At sufficiently low temperatures an orientational octupole glass can develop in a $\mathrm{Kr}-n \mathrm{CH}_{4}$ solution [14]. In our solutions with low $\mathrm{CH}_{4}$ concentrations ( $n \leq 10 \%)$ we can hardly expect an orientational glass to be formed due to a direct octupole interaction of $\mathrm{CH}_{4}$ molecules. Such concentrations may, however, allow an octupole glass caused by an indirect interaction. This type of glass can develop due to an indirect interaction of impurity molecules through the strain fields induced by the impurities themselves in the host lattice. The presence and type of glass can be identified from the temperature and concentration dependences of the heat capacity [12]. Quadrupole glasses with an indirect interaction have been detected and studied by several investigators [12-14].

\section{Experiment}

The heat capacity of solid $\mathrm{Kr}-n \mathrm{CH}_{4}$ solutions with the concentrations $n=1 ; 5 ; 10 \%$ and the $\mathrm{Kr}-1 \% \mathrm{CH}_{4}-0.2 \% \mathrm{O}_{2}$ solution were studied at $0.7-8 \mathrm{~K}$ by a pulse-heating method using an adiabatic vacuum calorimeter [15]. The temperature of the sample changed as $\Delta T_{i}=T_{i+1}-T_{i}$, where $T_{i}$ and $T_{i+1}$ are the temperatures before and after heating. The change during one measurement run was about $10 \%$ of $T_{i}$. The heat capacity corresponded to the temperature $T=\left(T_{i}+T_{i+1}\right) / 2$. The heating time $t_{h}$ was usually two or three minutes. The time $t_{\exp }$ taken to measure one heat capacity value was $0.5-1.4$ hour and depended mainly on the duration $\left(t_{e}\right)$ of temperature equalization over the sample after switching off the heater. The effective (characteristic) time $t_{m}$ of one measurement run is taken to be $t_{m}=t_{h}+t_{e}$. The mass of the samples was $0.5-0.7$ mole

The purity of the gases was: $\mathrm{CH}_{4}-99.94 \%$ $\left(0.04 \% \mathrm{~N}_{2}, \quad<0.01 \% \mathrm{Ar}, \mathrm{O}_{2}\right) ; \mathrm{Kr}-99.72 \%$ $\left(0.08 \% \mathrm{~N}_{2} ; 0.2 \% \mathrm{Xe}\right) ; \mathrm{O}_{2}-99.99 \%$.

Gas mixtures with a pre-assigned composition were prepared at room temperature. The impurity concentration and the mass of the samples were found from the $P V T$ data to within $0.2 \%$. A solid solution was prepared in a calorimeter at $T \approx 70 \mathrm{~K}$ by condensing the gas mixture into the solid phase. The error of the heat capacity measurement was $6 \%$ at $0.7 \mathrm{~K} ; 2 \%$ at $1 \mathrm{~K} ; 1 \%$ at $2 \mathrm{~K}$ and $0.5 \%$ above $4 \mathrm{~K}$. To minimize the systematic measurement errors and to improve the accuracy of separation of the heat capacity component $C_{\text {rot }}$ contributed by the $\mathrm{CH}_{4}$ impurity rotation, the heat capacity of pure $\mathrm{Kr}$ was measured in the same calorimeter.

The heat capacity of $\mathrm{Kr}-\mathrm{CH}_{4}$ solutions was measured only once, by A. Euken and H. Veight [9] in $1936\left(T=12-25 \mathrm{~K} ; 28-96 \% \mathrm{CH}_{4}\right)$.

\section{Data presentation}

The heat capacity of the $\mathrm{Kr}-\mathrm{CH}_{4}$ solution can be presented as a sum $C_{\text {sol }}=C_{\mathrm{tr}}+C_{\text {rot }}$, where $C_{\text {rot }}$ is the heat capacity contributed by the rotational motion of the $\mathrm{CH}_{4}$ molecules; $C_{\mathrm{tr}}$ is the heat capacity due to translational vibrations of a lattice. $C_{\mathrm{tr}}$ can in turn be represented as $C_{\mathrm{tr}}=C_{\mathrm{Kr}}+\Delta C_{L}$, where $C_{\mathrm{Kr}}$ is the heat capacity of pure krypton, and $\Delta C_{L}$ is the change in the translational heat capacity caused by the presence of a lighter impurity $\left(\mathrm{CH}_{4}\right)$ in the $\mathrm{Kr}$ lattice. $\Delta C_{L}$ was calculated by the Jacobi matrix method [16] for the mass ratio $m_{\mathrm{CH}_{4}} / m_{\mathrm{Kr}}=0.2$, the force constant variation being neglected. The rotational component $C_{\text {rot }}$ was found by subtracting $C_{\mathrm{Kr}}$ and $\Delta C_{L}$ from the experimental $C_{\text {sol }}$ values. The $C_{\text {rot }}$ value thus obtained was compared with calculation for three limiting cases:

1. Equilibrium distribution (ED) of the nuclear spin ( $A, E, T)$ modifications (fast conversion).

It is assumed that conversion is so fast that the $A, E$, and $T$ concentrations can be thought of as equilibrium at any instant at the temperature of the experiment. This is possible if conversion occurs during the effective time $t_{m}$ of a single measurement run. In this case the heat capacity is obtainable from the unified rotational energy spectrum based on all levels. 
2. Frozen equilibrium distribution (FED).

The $A, E$, and $T$ concentrations are equilibrium with respect to the initial temperature $T_{i}$ and do not change during the effective time $t_{m}$ when the sample is heated from $T_{i}$ to $T_{i+1}$. This is possible when the characteristic conversion time is $\tau>>t_{m}$. Prior to measurement, the sample must be kept at $T_{i}$ during the time $t>>$. At the mid-heating temperature $T=\left(T_{i}+T_{i+1}\right) / 2$, the heat capacity is calculated as

$$
C_{\text {rot }}(T)=x_{A}\left(T_{i}\right) C_{A}(T)+x_{T}\left(T_{i}\right) C_{T}(T)+x_{E}\left(T_{i}\right) C_{E}(T),
$$

where $x_{A}, x_{T}, x_{E}$ are the relative equilibrium $A, E$, and $T$ concentrations at $T_{i} ; C_{A}, C_{T}$, and $C_{E}$ are the heat capacities of the corresponding modifications at the temperature $T$.

3. Frozen distribution (FD) (no conversion).

The $A, E$, and $T$ concentrations are constant in the whole range of temperatures and equal to the hightemperature distribution, i.e., $x_{A}: x_{T}: x_{E}=5: 9: 2$. This occurs if at all temperatures the conversion is very slow and the $A, E$, and $T$ concentrations are practically invariable during the whole experiment (several weeks). In this case the heat capacity $C_{\text {rot }}$ can be found as

$$
C_{\text {rot }}(T)=\frac{5}{16} C_{A}(T)+\frac{9}{16} C_{T}(T)+\frac{2}{16} C_{E}(T) .
$$

To calculate $C_{\text {rot }}$ for the above limiting cases, we need to know the rotation spectrum of the $\mathrm{CH}_{4}$ molecule in the crystal field of krypton. This spectrum has been calculated in [4] (see Fig. 1,b). The energies of four low-laying rotational levels of the $\mathrm{CH}_{4}$ molecule in a $\mathrm{Kr}$ matrix were found experimentally by the method of inelastic neutron scattering $[5,17]$. It turns out that three of them agree well with theoretical predictions [4], the discrepancy being within 3\% (see Fig. 1,c). The fourth level differs by about $30 \%$. We thought it reasonable to use the spectrum of Fig. $1, b$ in the subsequent $C_{\text {rot }}$ calculation.

\section{Results of the measurements. Discussion}

The heat capacities $C_{\text {rot }}$ measured on the solutions with $1 ; 5 ; 10 \% \mathrm{CH}_{4}$ and normalized to the $\mathrm{CH}_{4}$ concentration $n$ and the universal gas constant $R$ are shown in Fig. 3, $a$ for the whole range of measurement temperatures and in Fig. 3,b (enlarged scale) up to $4.3 \mathrm{~K}$. As seen in Fig. 3,a, the data scatter increases with rising temperature, and the accuracy of the $C_{\text {rot }}$ component degrades. This is due to the lattice contribution $C_{\mathrm{tr}}$ to the total heat capacity of the solution, which increases when the temperature rises: $47 \%$ at $T=4 \mathrm{~K}$ and $78 \%$ at $T=8 \mathrm{~K}(n=10 \%)$. The dotted line in Fig. 3, $a$ corresponds to the high-temperature value, $C_{\text {rot }} / n R=3 / 2$, for a mole of isolated $\mathrm{CH}_{4}$ molecules in a $\mathrm{Kr}$ matrix.

Along with experimental results, Fig. 3 show the calculated heat capacities for the above three limiting cases: ED (curve 1), FED (curve 2), FD (curve 3). It is seen that in the whole temperature region, the heat capacity of the $\mathrm{Kr}-1 \% \mathrm{CH}_{4}$ solution hardly exceeds the experimental error and is much below curves 1 (ED) and 3 (FD) at $T \leq 5 \mathrm{~K}$. Curve 2 (FED) is the best description of the solution at $T \leq 5 \mathrm{~K}$. This suggests that the characteristic conversion times of this solution greatly exceed the effective times $t_{m}$ of a single measurement run. This conclusion is consistent with $[18,19]$. According to [18] the characteristic conversion time $\tau$ of isolated $\mathrm{CH}_{4}$ molecules in a $\mathrm{Kr}$ matrix is 3.5 hours at $T=2 \mathrm{~K}$.
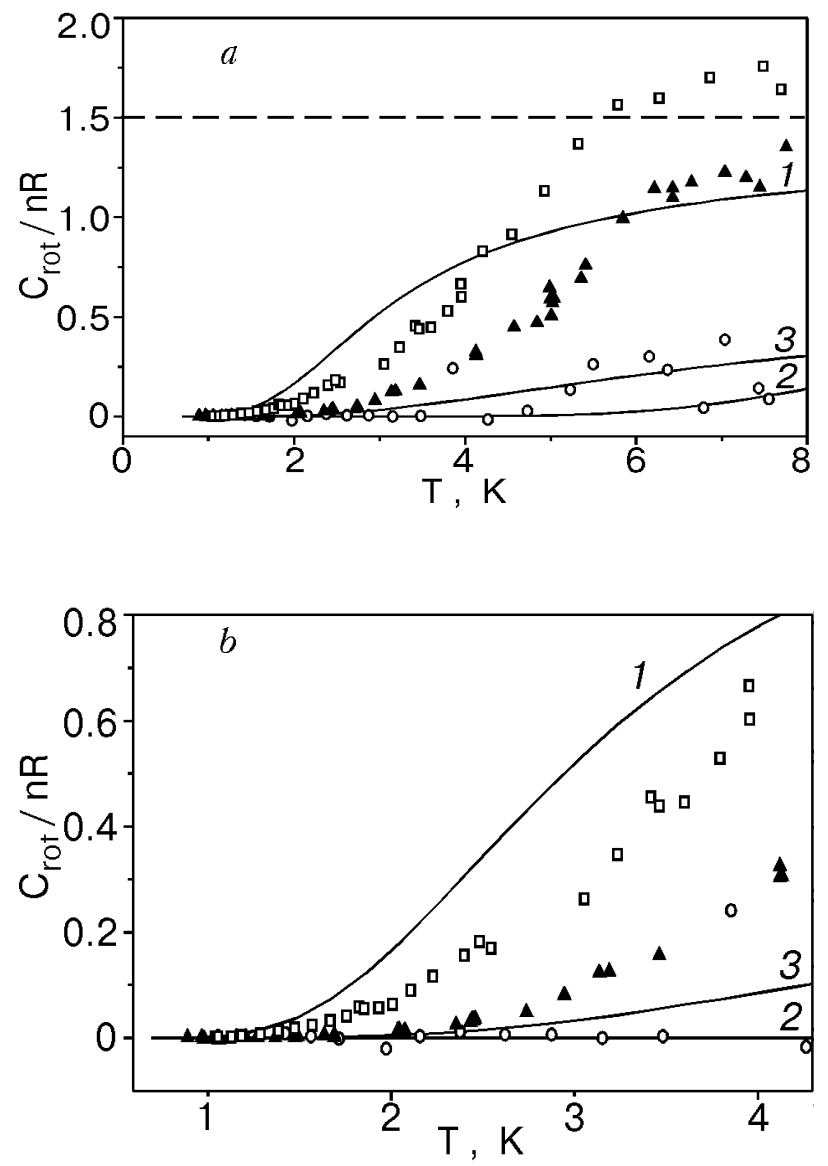

Fig. 3. Temperature dependence of reduced rotational heat capacity of $\mathrm{Kr}-\mathrm{CH}_{4}$ solutions containing $1 \% \mathrm{CH}_{4}(\mathrm{O}), 5 \% \mathrm{CH}_{4}$ $(\Delta), 10 \% \mathrm{CH}_{4}(\square)$ for the whole temperature range $(a)$ and up to $4.3 \mathrm{~K}(b)$. The solid lines are the theoretical curves for the three limiting cases mentioned ( 1 - ED, 2 - FED, 3 - FD). 
It is seen in Fig. 3 that the reduced rotational heat capacity increases rapidly with the concentration $n$, and above $T \approx 4 \mathrm{~K}(n=10 \%)$ or $T \approx 6 \mathrm{~K}$ ( $n=5 \%$ ) it exceeds the $C_{\text {rot }} / n R$ value corresponding to the instantaneous thermodynamic equilibrium of the nuclear spin modifications of isolated $\mathrm{CH}_{4}$ molecules. This can be explained as follows. As the concentration $n$ grows, the number of $\mathrm{CH}_{4}$ clusters increases, too. Clusters of two and three $\mathrm{CH}_{4}$ molecules prevail at $n=5 ; 10 \% \mathrm{CH}_{4}$. The hybrid mechanism of conversion operating in the clusters [6] enhances the conversion rate considerably. As a result, the nonequilibrium nuclear spin modifications come into equilibrium, and the entropy $S$ of the solution increases. The higher the conversion rate is in the nonequilibrium system, the more intensively the entropy changes at the initial temperature $T_{i}$ during the effective time $t_{m}$ of a single measurement run. Correspondingly, the measured heat capacity $C_{V}=T(d S / d T)$ appears to be higher. Note that in this case the measured heat capacity can exceed that of the equilibrium system. The higher heat capacity of clustered molecules (as compared to that of isolated ones) can also contribute appreciably to the concentration dependence of the heat capacity. We should not therefore overestimate the quantitative comparison between the $C_{\text {rot }}$ of the solutions with $n=5 ; 10 \% \mathrm{CH}_{4}$ and the theoretical curve describing the rotational heat capacity of the equilibrium system of isolated impurity molecules (the ED case).

Additional arguments for or against the enhanced conversion in clusters can be furnished by the consequences that follow from this assumption. Let us assume that (i) the contribution of isolated $\mathrm{CH}_{4}$ molecules (singles) to the heat capacity is negligible, (ii) the conversion rate in a cluster is independent of its size, and (iii) the rotational energy spectrum of the molecules in clusters is also independent of the cluster sizes. If these conditions are realized, the reduced heat capacity $C_{\text {rot }} / n^{\prime} R$, where $n^{\prime}$ is the molecule concentration in the clusters, will be independent of the methane concentration $n$. And we remember that the number of $\mathrm{CH}_{4}$ singles in a solution containing $N$ particles is $N n(1-n)^{12}$; correspondingly, the number of molecules in clusters is $N n\left[1-(1-n)^{12}\right]$, i.e., $n^{\prime}=$ $=n\left[1-(1-n)^{12}\right]$.

In Fig. 4 the heat capacity of the rotational subsystem in the solutions with $5 \% ; 10 \% \mathrm{CH}_{4}$ is shown in the reduced coordinates $C_{\text {rot }} / R n\left[1-(1-n)^{12}\right]-T$. It is seen that the reduced heat capacities of these solutions coincide at 5-8 $\mathrm{K}$ within the measurement error, i.e., they

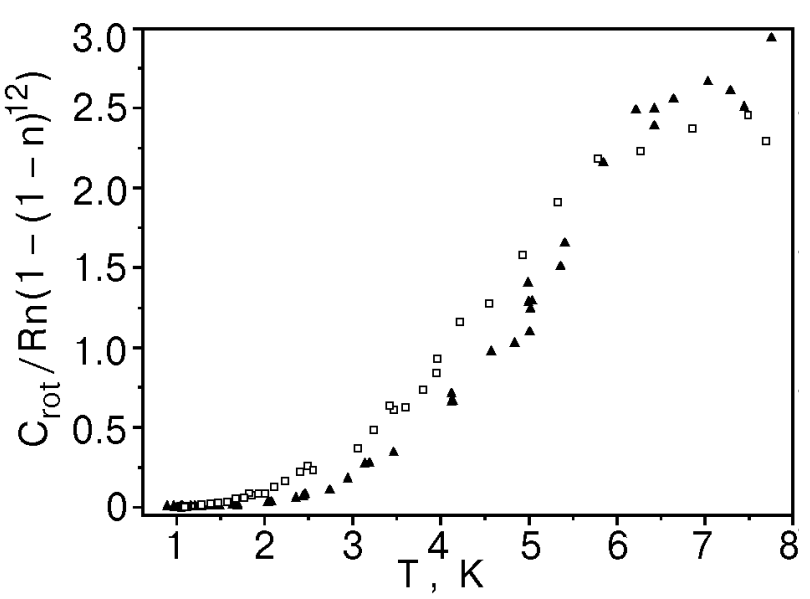

Fig. 4. Temperature dependence of rotational heat capacity of $\mathrm{Kr}-\mathrm{CH}_{4}$ solutions containing $5 \% \mathrm{CH}_{4}(\boldsymbol{\Delta}), 10 \% \mathrm{CH}_{4}(\square)$ in reduced coordinates.

are independent of the methane concentration. This supports the assumption of considerably enhanced conversion in clusters and suggests that the above three conditions are realized in this temperature interval.

At lower temperatures (see Fig. 4) there are some deviations from the universal dependence which exceed the experimental error. This may occur because conditions (ii) and (iii) (or one of them) are not fulfilled. According to [3], the first effective excited rotational level $(J=1)$ is somewhat lower for three-molecule clusters $(i=3)$ than for two-molecule ones $(i=2)$. Correspondingly, at rather low temperatures the heat capacity of the solution with $n=10 \%$, which contains more $i=3$ clusters, should be appreciably higher than in the $n=5 \%$ case. Another reason may be connected with the conversion rate, which becomes dependent on the cluster size as the temperature is lowered.

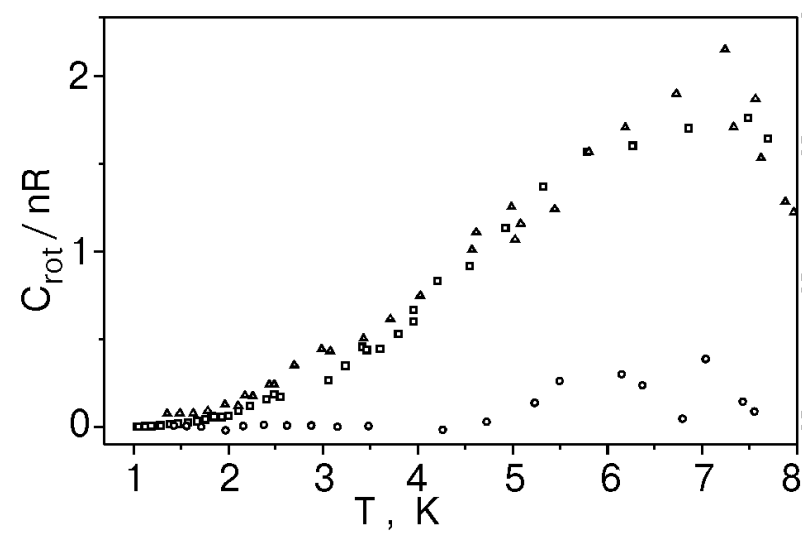

Fig. 5. Temperature dependence of reduced rotational heat capacity of binary $\mathrm{Kr}-\mathrm{CH}_{4}$ solutions containing $1 \% \mathrm{CH}_{4}(\mathrm{O})$, $10 \% \mathrm{CH}_{4}(\square)$ and the triple solution $\mathrm{Kr}-1 \% \mathrm{CH}_{4}-0.2 \% \mathrm{O}_{2}$ $(\Delta)$. 


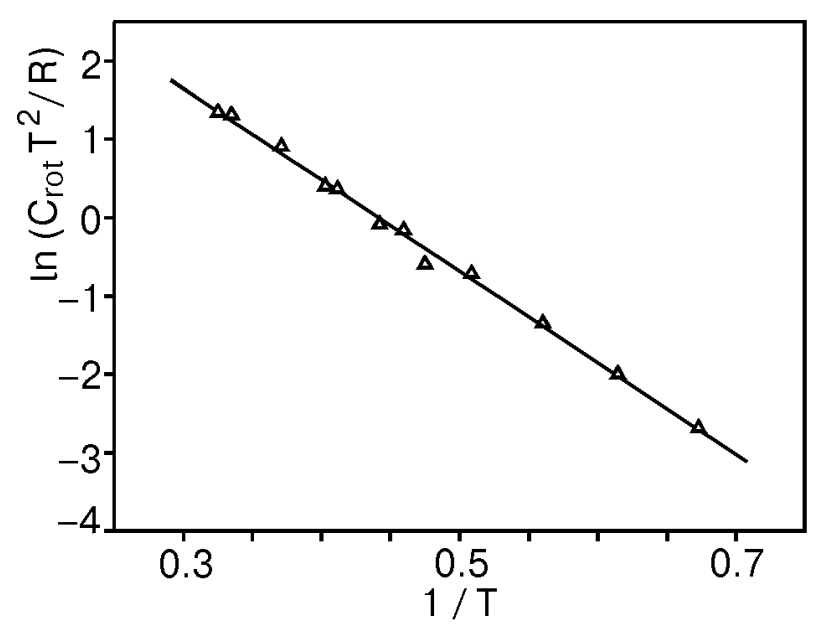

Fig. 6. Temperature dependence of $\mathrm{C}_{\text {rot }}$ of the $\mathrm{Kr}-1 \% \mathrm{CH}_{4}-$ $0.2 \% \mathrm{O}_{2}$ solid solution in $\ln \left(C_{\text {rot }} T^{2} / R\right)-1 / T$ coordinates.

To estimate the effect of a paramagnetic impurity on the conversion rate and hence on the heat capacity, we investigated the heat capacity of the $\mathrm{Kr}-1 \% \mathrm{CH}_{4}-0.2 \% \mathrm{O}_{2}$ solution. The contributions of the impurities $\left(\mathrm{CH}_{4}, \mathrm{O}_{2}\right)$ were assumed to be additive. The contribution of $\mathrm{O}_{2}$ to the heat capacity of the solution was estimated using the data of [20]. In the presence of $\mathrm{O}_{2}$ the $\mathrm{CH}_{4}$ contribution to the heat capacity is an order of magnitude higher than that in the $\mathrm{Kr}-1 \% \mathrm{CH}_{4}$ solution containing no appreciable paramagnetic impurities (see Fig. 5).

With an equilibrium distribution of the $\mathrm{CH}_{4}$ modifications, the heat capacity at $T \leq 3 \mathrm{~K}$ is determined by the position of the first excited level in the rotational spectrum of the $\mathrm{CH}_{4}$ molecules (see Fig. 1). We assume that at $T \leq 3 \mathrm{~K}$ some part of the $\mathrm{CH}_{4}$ molecules influenced by the paramagnetic impurities convert so fast that their heat capacity corresponds to the equilibrium distribution (the ED case). Let this fraction of molecules be denoted as $n^{\prime \prime}$. The value of $n^{\prime \prime}$ also includes a relatively small number of the fast-converting $\mathrm{CH}_{4}$ molecules from the clusters. We then assume that the contribution of the rest of the $\mathrm{CH}_{4}$ molecules, whose fraction is $\left(1-n^{\prime \prime}\right)$, to $C_{\text {rot }}$ is negligible. When the above three conditions are fulfilled, $C_{\text {rot }}$ can be written as

$$
C_{\mathrm{rot}}=N k_{B} \frac{n^{\prime \prime}}{T^{2}} E_{1}^{2} \frac{g_{1}}{g_{0}} \mathrm{e}^{-E_{1} / T},
$$

where $N$ is the number of $\mathrm{CH}_{4}$ molecules in the sample; $E_{1}$ is the first excited level energy; $g_{0}$ and $g_{1}$ are the degeneracies of the ground and the first excited levels, respectively; $k_{B}$ is Boltzmann's constant. In Fig. 6 the measured $C_{\text {rot }}$ of the $\mathrm{Kr}-1 \% \mathrm{CH}_{4}-0.2 \% \mathrm{O}_{2}$ solution is shown in the coordinates $\ln \left(C_{\text {rot }} T^{2} / R\right)-1 / T$.

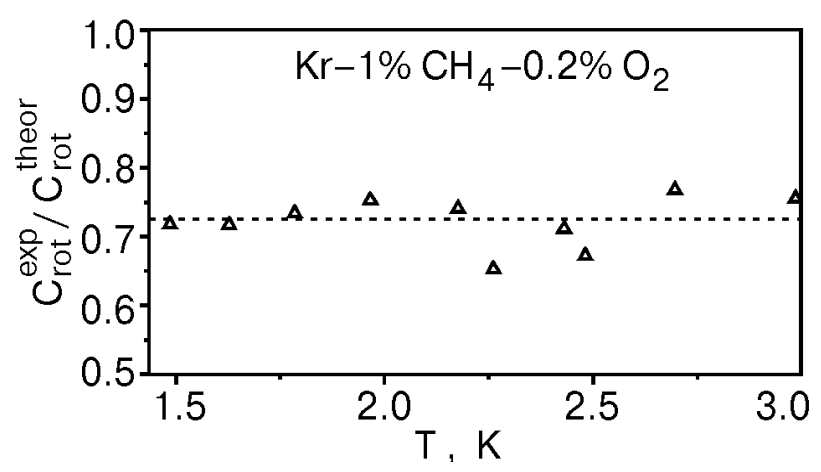

Fig. 7. Temperature dependence of the ratio of $C_{\text {rot }}^{\exp }$ to $C_{\text {rot }}^{\text {theor }}$ for $E_{1}=11.7 \mathrm{~K}$.

The linear dependence $\ln \left(C_{\text {rot }} T^{2} / R\right)-1 / T$ is fulfilled within the experimental error, which indicates that our assumptions are realistic. The $E_{1}$ value $(11.7 \pm 0.4 \mathrm{~K})$ was estimated from the slope of the obtained straight line and is in good agreement with the neutron-diffraction magnitude $E_{1}=11.7 \mathrm{~K}$ [17].

The ratio of the experimental $C_{\mathrm{rot}}^{\exp }$ and the $C_{\text {rot }}^{\text {theor }}$ calculated for $E_{1}=11.7 \mathrm{~K}$ on the assumption that all the $\mathrm{CH}_{4}$ molecules convert during the effective time $t_{m}$ of a single heat capacity measurement (the ED case) is shown in Fig. 7. It is seen that $n^{\prime \prime}=72 \%$, which implies that only $72 \%$ of $\mathrm{CH}_{4}$ molecules convert in the $\mathrm{Kr}-1 \% \mathrm{CH}_{4}-0.2 \% \mathrm{O}_{2}$ solid solution at $T \leq 3 \mathrm{~K}$ during the effective time $t_{m}$ of a single heat capacity measurement. This suggests that during the time $t_{m}$ the conversion takes place in the $\mathrm{CH}_{4}$ molecules which are situated in seven coordination spheres around the $\mathrm{O}_{2}$ molecule.

Finally, we discuss the possibility of an orientational glass in our $\mathrm{Kr}-\mathrm{CH}_{4}$ solutions. At the $\mathrm{CH}_{4}$ concentrations used in this study, we can only expect the orientational glass with an indirect interaction [12]. In such glasses the rotators interact through the strain fields which they form in the matrix. Our temperature and concentration dependences do not display any features typical for the heat capacity of such glasses [12]. This means that the shift of the $\mathrm{CH}_{4}$ rotation energy levels caused by the indirect interaction of the $\mathrm{CH}_{4}$ molecules is smaller than the distance $E_{1}$ between the ground and the first excited levels of the spectrum. It is quite possible that the glass-like behavior should be sought in the temperature and concentration dependences of $\mathrm{CD}_{4}-\mathrm{Kr}$ solid solutions because $E_{1}$ is smaller in that case.

The authors are indebted to E. S. Syrkin, S. B. Feodosiev, and I. A. Gospodarev for the calculated data on the translational component of the heat 
capacity of $\mathrm{Kr}-\mathrm{CH}_{4}$ solutions. This study has been supported by the Ministry of Education and Science of Ukraine within the Project «Optical and Thermal Spectroscopy of Small Molecules in Cryogenic Matrices» No. M/1862-97.

1. Physics of Cryocrystals, V. G. Manzhelii and Yu. A. Freiman (eds.), AIP press, Woodbury, New York (1997).

2. G. Herzberg, Infrared and Raman Spectra of Polyatomic Molecules, New York (1945), p. 647.

3. T. Yamamoto, Y. Kataoka, and K. Okada, J. Chem. Phys. 66, 2701 (1977).

4. K. Nishiyama and T. Yamamoto, J. Chem. Phys. 58, 1001 (1973).

5. B. Asmussen, M. Prager, W. Press, H. Blank, and C. J. Carlile, J. Chem. Phys. 97, 1332 (1992).

6. A. J. Nijman and A. J. Berlinsky, Canad. J. Phys. 58, 1049 (1980).

7. I. M. Lifshits, Zh. Eksp. Teor. Fiz. 26, 551 (1954).

8. A. I. Krivchikov, M. I. Bagatskii, V. G. Manzhelii, I. Ya. Minchina, and P. I. Muromtsev, Fiz. Nizk. Temp. 14, 1208 (1988) [Sov. J. Low Temp. Phys. 14, 667 (1988)].

9. A. Euken and H. Veith, Z. Phys. Chem. B34, 275 (1936).

10. H. Veith and E. Schrouder, Z. Phys. Chem. A179, 16 (1937).
11. F. de Luca and B. Maraviglia, Phys. Lett. 101, 300 (1983).

12. V. G. Manzhelii, M. I. Bagatskii, I. Ya. Minchina, and A. N. Aleksandrovskii, J. Low. Temp. Phys. 111, 290 (1998).

13. A. I. Burin, Fiz. Nizk. Temp. 17, 872 (1991) [Sov. J. Low Temp. Phys. 17, 456 (1991)].

14. M. I. Bagatskii, V. G. Manzhelii, M. A. Ivanov, P. I. Muromtsev, and I. Ya. Minchina, Fiz. Nizk. Temp. 18, 1142 (1992) [Low Temp. Phys. 18, 801 (1992)].

15. M. I. Bagatskii, I. Ya. Minchina, and V. G. Manzhelii, Fiz. Nizk. Temp. 10, 1039 (1984) [Sov. J. Low Temp. Phys. 10, 542 (1984)].

16. V. I. Peresada and V. P. Tolstoluzhskii, Fiz. Nizk. Temp. 3, 788 (1977) [Sov. J. Low Temp. Phys. 3, 378 (1977)].

17. B. Asmussen, W. Press, N. Prager, and H. Blank, J. Chem. Phys. 98, 158 (1993)

18. S. G. Grieger, H. Friedrich, B. Asmussen, K. Guckelsberger, D. Nettling, W. Press, and R. Scherm, Z. Phys. B. Condens. Matter 89, 203 (1992).

19. V. V. Dudkin, B. Ya. Gorodilov, A. I. Krivchikov, and V. G. Manzhelii, Fiz. Nizk. Temp. 26, 1023 (2000) [Low Temp. Phys. 26, 762 (2000)].

20. P. I. Muromtsev, M. I. Bagatskii, V. G. Manzhelii, and I. Ya. Minchina, Fiz. Nizk. Temp. 20, 247 (1994) [Low Temp. Phys. 20, 195 (1994)]. 